\title{
Review of skull base chordomas: prognostic factors and long-term results of proton-beam radiotherapy
}

\author{
EugEN B. Hug, M.D. \\ Department of Radiation Oncology, Dartmouth Hitchcock Medical Center, Lebanon, New Hampshire
}

\begin{abstract}
Fractionated proton beam radiotherapy has been used for skull base tumors in the United States since the mid-70s, and more than 300 patients in whom diagnosis of chordoma of the skull base has been made have been treated. The ability to achieve high degrees of radiation dose conformity by using protons has resulted in higher radiation doses than can be delivered with conventional radiotherapy in the base of skull. High target volume doses have led to improved tumor control and patient survival. Side effects such as severe toxicity are acceptable considering the alternatives of uncontrolled tumor growth. The authors of various analyses have identified prognostic factors that can be used to predict a patient's chance of treatment success. On the horizon are important technical developments that will further increase dose conformity and increase target doses. In this paper the author reviews long-term outcome data and prognostic predictors for survival of patients with skull base chordomas based on the largest worldwide patient series.
\end{abstract}

KEY WORDS • chordoma • proton • radiation therapy • skull base tumor

In 1974 the first patient presenting with skull base chordoma underwent proton irradiation at MGH/HCL. Following a first report by Austin-Seymour, et al., ${ }^{2}$ patient referral steadily increased. Collaborators at LBNL have treated patients with skull base tumors since 1977, but subsequently LBNL ceased its operation. In 1990, the hospital-based proton radiotherapy facility at LLUMC opened. At present, the two proton radiotherapy facilities in the United States, MGH/HCL and LLUMC, have accumulated the largest worldwide patient population treated for skull base chordomas. Treatment philosophies at both centers are similar and treatment protocols are shared.

Compared with other forms, photon radiation has the advantage of the physical properties of dose deliverythat is, dose deposition within the tumor by using a spread-out Bragg peak with sharp dose falloff downstream. Because protons do not have an exit dose, dose distributions can be designed that are highly conformal in all three dimensions. Protons are highly adaptable to irregular target volumes, and this makes them particularly useful in the skull base where tumors are likely of irregular

Abbreviations used in this paper: $\mathrm{CGE}=$ cobalt gray equivalent; GKS = gamma knife surgery; LBNL = Lawrence Berkeley National Laboratory; LINAC = linear accelerator; LLUMC = Loma Linda University Medical Center; MGH/HCL = Massachusetts General Hospital/Harvard Cyclotron Laboratory. contour and in close proximity to vital normal structures. Protons are assumed to have a slightly higher relative biological effectiveness compared with photons. A clinical working factor (relative biological effectiveness factor) of 1.1 is used in both centers to adjust the proton dose for its higher biological effectiveness. All proton data reported in this review are based on a course of fractionated radiotherapy at 1.8- to 2.0-CGE) dose per fraction. At LLUMC the majority of patients are treated exclusively with proton beam radiotherapy (five treatments per week). At MGH/ HCL the proton beam system is unavailable 1 workday per week. Thus photon therapy is substituted for proton beam once per week resulting in a mixed proton-photon course (photon component $<30 \%$ of the overall radiation dose).

Having accumulated a sufficient number of patients and an adequate follow-up period, collaborators at MGH/ HCL, LBNL, and LLUMC were able to identify clinical variables predictive of long-term tumor control. In this review long-term outcome data and prognostic indicators are summerized.

\section{LONG-TERM OUTCOME AND COMPLICATIONS}

Between 1977 and 1992 at LBNL, 126 patients with skull base tumors underwent charged particle (helium or 


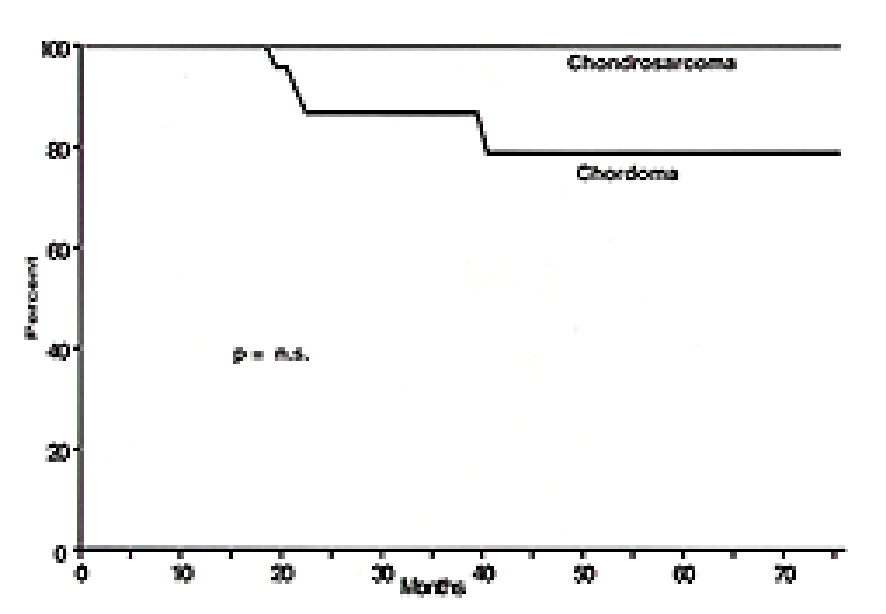

Fig. 1. Graph showing overall survival following fractionated proton irradiation for skull base chordomas (33 patients) and chondrosarcomas (25 patients).

neon ions) therapy. ${ }^{6}$ Follow-up time ranged from 4 to 201 months (median 53 months), and radiation doses ranged from 60 to 80 CGE (mean 68 CGE). Fifty-three patients were treated for skull base chordomas. The 5-year local control and overall survival rates were $63 \%$ and $75 \%$, respectively. In a separate analysis of 45 patients, complications in the LBNL series included three cases of unilateral loss of vision, two of blindness, and four cases of brainstem injury $(17 \%){ }^{4}$

The MGH/HCL group recently published a report on 621 patients with skull base and cervical chordomas or chondrosarcomas treated since $1975 .^{14}$ The 519 cases of skull base tumor included 290 chordomas (159 male and 131 female patients) and 229 low-grade chondrosarcomas. Prescribed doses ranged from 66 to 83 CGE, when using a proton relative biological effectiveness value of 1.1. Daily doses were 1.8 or 1.92 CGE, respectively, given 5 days per week usually in four fractions of protons and one of megavoltage x-rays. With increasing follow-up time (median 41 months; maximum almost 22 years) and increasing number of patients, a significant difference in local control and survival rates was demonstrated between patients with chondrosarcomas and those with chordomas. At 5 and 10 years, the local recurrence-free survival rates were $97 \%$ and $92 \%$, respectively for chondrosarcomas, and $64 \%$ and $42 \%$, respectively, for chordomas. Analysis of the $\mathrm{MGH} / \mathrm{HCL}$ data strongly suggested that low-grade chondrosarcomas are a clinically distinct entity from chordomas. Severe toxicities were reported in $8 \%$ of patients, including 12 cases in which there were symptomatic or asymptomatic brain changes, three cases of unilateral and two cases of bilateral blindness, and four cases of unilateral deafness. ${ }^{15}$

In 1999, our group reported outcome data obtained in 58 patients treated at LLUMC for low-grade skull base chordomas and chondrosarcomas. ${ }^{10}$ Thirty-three patients with chordomas and 25 patients with chondrosarcomas had undergone fractionated proton beam radiotherapy to target doses ranging from 64.8 to 79.2 CGE (mean 70.7 CGE). The dose per fraction was 1.8 CGE one fraction per day 5 days per week. More than $90 \%$ of patients un- derwent proton irradiation exclusively. In a mean followup period of 33 months (range 7-75 months) eight (24\%) of 33 patients with chordomas experienced recurrence compared with two (8\%) of 25 patients with chondrosarcoma in whom the tumor recurred. Thus, in $76 \%$ of patients with chordomas and in $82 \%$ of patients with chondrosarcomas, local tumor control was achieved. On actuarial analysis, these data resulted in local control rates at 3 years of $94 \%$ for patients with chondrosarcomas and $67 \%$ for patients with chordomas. Five-year actuarial overall survival rates were $79 \%$ for patients with chordomas and $100 \%$ for patients with chondrosarcomas (Fig. 1). Grade 3 and 4 late toxicities were observed in four patients (7\%) and were symptomatic in three patients $(5 \%)$.

\section{PROGNOSTIC FACTORS}

\section{Predictive Value of the Size and/or Location of Gross or Residual Disease}

Proton beam radiotherapy has no technical size limitations. In fact, most tumors treated with proton irradiation routinely exceed the size limitations imposed in GKS. ${ }^{11}$ Data accrued at LLUMC were analyzed according to tumors with volumes less than/equal to or greater than 25 $\mathrm{ml} .{ }^{10}$ Thus, based on size, one group of tumors was potentially suitable for GKS and the other (exceeding that size) was not. In all patients with preirradiation tumor volume not exceeding $25 \mathrm{ml}$ local tumor control was achieved, whereas in $56 \%$ of those with larger-volume tumors $(>25$ $\mathrm{ml})$ tumor control was achieved $(\mathrm{p}=0.02)$ (Fig. 2). At LBNL, tumors were classified as small $(<20 \mathrm{ml})$, intermediate $(20-35 \mathrm{ml})$ or large $(>35 \mathrm{ml}){ }^{4}$ Significantly higher local control rates (80\% compared with 33\%, respectively) were observed for smaller volumes. This analysis included patients with chordomas and chondrosarcomas. In an MGH/HCL review the authors documented a significant disease-specific survival difference only in patients with large $(<70 \mathrm{ml})$ compared with very large tumors $(>70 \mathrm{ml}) .^{15}$

In choosing the appropriate treatment modality, the location of any residual gross tumor is at least equally important to the actual size of the tumor. A midclival tumor that extends into the nasopharynx or sphenoid sinus, but without intracranial extension, is amenable to various radiation treatments. However, if the midclival tumor extends intracranially and abuts or compresses the brainstem or displaces the optic chiasm, the radiation dose deliverable to the tumor component in immediate contact with these critical structures will be limited. Photon radiosurgery and proton radiation therapy can be used to create a sharp dose gradient in an effort to minimize the volume of tumor not receiving the full, prescribed, tumoricidal dose. Fractionated radiotherapy has the added benefit of sublethal damage repair of normal tissues in between treatment fractions, thus resulting in higher critical normal tissue tolerance levels. The importance of resecting tumor from critical normal tissues has been documented by the LLUMC group. ${ }^{10}$ In 59\% of their patients brainstem abutment or compression was present prior to proton irradiation. Local control rates at 5 years were $94 \%$ in cases without and $53 \%$ in cases with brainstem involvement $(p=0.04)$. Austin, et al., ${ }^{1}$ reported for the MGH/HCL 


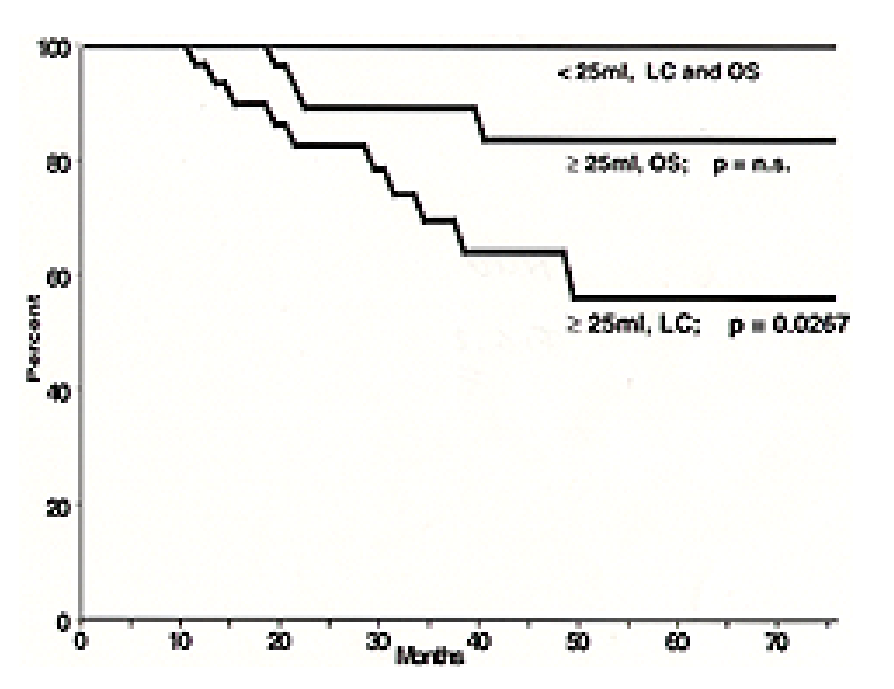

Fig. 2. Graph depicting local control (LC) and overall survival (OS) following fractionated proton irradiation for skull base chordomas (33 patients) and chondrosarcomas (25 patients) according to preirradiation gross tumor volume. NS = not significant.

group that the majority of treatment failures (in 15 of 26 patients) after radiotherapy occurred in a region of the tumor that had received a suboptimum radiation dose because of the lesion's proximity to surrounding normal tissues.

In summary, analysis of available data indicates a local control and survival benefit, in previously resected largeor small-sized tumors if they can be debulked and freed from critical normal structures.

\section{Relation of Higher Radiation Doses to Improved Outcome}

In conventional radiotherapy the radiation dose to the tumor is limited according to the maximum dose that nearby critical normal tissues can tolerate (for example, brainstem optic nerves and optic chiasm). This strategy has resulted historically in fractionated total radiation doses ranging from 50 to 60 Gy. Rarely was a dose of 60 or 65 Gy exceeded. Recurrence rates as high as 50 to $100 \%$ have been reported after conventional megavoltage irradiation. Local control and survival curves follow a continued downward slope. ${ }^{7,8,17,19}$ Proton radiotherapy in which the authors applied mean doses of $71 \mathrm{CGE}$ (LLUMC series) and doses up to 83 CGE (MGH/HCL series) has resulted in increased tumor control. Almost all patients undergoing proton radiotherapy received doses greater than 66 CGE. ${ }^{11}$ In a multiinstitutional study, involving $\mathrm{MGH} / \mathrm{HCL}, \mathrm{LBNL}$, and later LLUMC, the investigators divided patients into low-risk and high-risk groups. Low risk cases were defined as male or female patients with chordomas, as well as male patients with skull base chondrosarcomas; high-risk cases were female patients with skull base chordomas, as well as male and female patients with cervical chordomas and chondrosarcomas. Patients in the low-risk group were randomly assigned to receive treatment with either 69.6 CGE or 75.6 CGE; patients in the high-risk group were randomly assigned to receive treatment with either 75.6 CGE or 82.9 CGE. Overall 328 patients were enrolled. ${ }^{14}$ This is the largest randomized study ever conducted in patients with skull base chordomas and chondrosarcomas. An interim report is expected soon.

\section{Predictive Value of Age or Sex on Increased Biological Aggressiveness}

In a limited analysis of 62 patients treated at $\mathrm{MGH} /$ HCL, an insignificant trend for patients older then 40 years of age was observed. ${ }^{13}$ In these patients worse disease-specific survival was demonstrated.

Recently, Al-Mefty, collaborating with Borba and colleagues, ${ }^{5}$ suggested that chordomas behave more aggressively in the pediatric population. For the $\mathrm{MGH} / \mathrm{HCL}$ group, Benk, et al., ${ }^{3}$ reported 18 pediatric patients in whom tumors were treated with doses ranging from 55.8 to $75.6 \mathrm{CGE}$. The 5-year actuarial overall survival rate was $68 \%$ and the local recurrence-free survival rate $78 \%$. The author treated 10 pediatric patients with skull base chordomas between 1992 and early 1999 at MGH/HCL and LLUMC. ${ }^{12}$ Patient age ranged from 2 to 19 years at time of diagnosis. The average prescribed tumor dose was 74 CGE (range 70-79 CGE). Local tumor control was maintained in six $(60 \%)$ of 10 patients. The author of the present report is aware of three children who developed and subsequently died of rapidly progressive metastatic disease. In general, it is the author's experience that chordomas in the pediatric patients behave more aggressively than in adults, with relatively short natural history and sometimes dramatic pace of tumor recurrence following initial resection.

The MGH/HCL group, with its large database, has early on reported a sex-specific significant difference in progression-free and disease-specific survival rates. In repeated updates, female patients with chordomas fared significantly and consistently worse than male patients. ${ }^{13-15}$ At 5 years posttreatment, actuarial tumor control was $81 \%$ in male and $65 \%$ in female patients, and at 10 years it was $62 \%$ and $42 \%$, respectively $(p=0.007)$. The tumor control curve for male patients appears to reach a plateau with a few recurrences developing after 10 years. In contrast, female patients continue to experience late recurrences. A trend toward female sex as a potentially negative prognostic predictor was also found in the LLUMC series, although it was not statistically significant (local tumor control at 5 years was $54 \%$ for female patients and $70 \%$ for male patients). ${ }^{10}$ In the author's series of pediatric patients with skull base tumors, all five girls with chordomas suffered tumor recurrence whereas only one of five boys experienced recurrence. ${ }^{12}$ The cause for this sex difference is unknown. Preliminary estrogen and progesterone receptor studies in adult patients were thus far inconclusive.

\section{Effects of Delayed Proton Irradiation on Tumor Control}

Unfortunately, in the largest series of patients (those treated at $\mathrm{MGH} / \mathrm{HCL}$ ), the authors did not include the variable of treatment for primary compared with recurrent disease in any recent analysis. Previously, the LBNL had found a higher 5-year local control rate for patients undergoing helium or neon radiotherapy at the time of initial diagnoses $(78 \%)$ compared with recurrent disease $(33 \%){ }^{6}$ No difference in outcome was demonstrated in the 
LLUMC study in patients undergoing radiotherapy for recurrent or primary disease.

A diagnosis of chordoma spans a wide variety of natural history and range of biological aggressiveness. In patients with documented several-year histories of slowly progressive symptoms, a policy of watchful observation following initial resection appears sometimes justified. Our knowledge of the true rate of long-lasting tumor control following modern microsurgical, gross-total resection is limited. Thus, it is difficult to develop general treatment recommendations.

Although currently we have limited evidence that delayed radiation treatment will effect the overall outcome negatively, it has to be kept in mind that tumor regrowth might occur at an unfavorable location, causing potentially irreversible neurological nerve damage. Additionally, repeated resections limit the tolerance of normal tissue to definitive high-dose proton irradiation. Debus, et al., ${ }^{9}$ have reported that repeated surgical procedures are an independent prognosticator for severe brainstem toxicity after proton radiotherapy.

\section{DISCUSSION}

In using proton radiation, practitioners have pioneered the concept of high-dose fractionated radiotherapy for skull base tumors. Three-dimensional conformal irradiation in the base of skull was also introduced by users of proton beam radiotherapy. Radiation doses in excess of 70 Gy, previously considered unsafe, were safely achieved, and valuable information on partial organ tolerance doses obtained.

Superior local tumor control and overall survival achieved using fractionated proton irradiation, compared with conventional photon radiotherapy, are attributed to superior dose localization characteristics of protons that result in higher doses delivered.

Over the past decades, we have witnessed a revolution in the application of photon radiotherapy. Rapid advancements in computer software as well as hardware have resulted in fractionated stereotactic radiotherapy (intensity-modulated radiation therapy) and LINAC-based radiosurgery. Thus, the ability to deliver a conformal photon radiation dose has greatly improved. In addition, indications for GKS have been expanded to include use in malignant skull base lesions. For small tumors with some distance from normal critical structures, there appears to be little difference between high-dose isodose target coverage between three-dimensional conformal photons and similar proton techniques. However, in larger lesions in immediate proximity to normal critical structures and in highly irregularly shaped tumors, proton radiotherapy has consistently been demonstrated, in comparisons with other modalities in terms of target planning and delivery, to maintain an advantage..$^{20}$ The fact remains that proton irradiation does not have an exit dose. Therefore, the integral volume of normal tissues receiving radiation will always be smaller compared with that when using photons. This is of particular importance in the pediatric patient in whom even small to moderate amounts of radiation can lead to major cosmetic and life-long functional impairments. A principal, philosophical difference exists be- tween single-fraction radiosurgery and fractionated proton irradiation: in general, only gross tumor volume and a limited microscopic target volume are targeted with either single or multiple isocenters in radiosurgery. Any additional microscopic disease beyond this target will be irradiated concentrically in the dose falloff region. In contrast, in using proton irradiation, it has been always our policy to identify separately a clinical target volume, with distinctly different shape and size compared with the gross tumor volume. This clinical target volume is defined as any area of risk for microscopic disease, in general including the entire operative bed and anatomical compartment of initial extension. The clinical target volume will receive a separately prescribed microscopic target dose in fractionated fashion. This concept has proven to be successful because the majority of any failures after proton radiotherapy occur within the gross tumor target volume itself and not in the operative bed.

The routine use of postoperative radiotherapy is controversial. To judge the potential benefits of adjuvant radiation therapy, we need to accrue long-term data on permanent tumor control rates since the introduction of modern microsurgical techniques to achieve total or near-total tumor resection.

In dose-escalation trials charged particle therapy continues to be studied. We await the results of the recent randomized study in which doses up to 83 CGE were used. Developments in proton radiotherapy include intensitymodulated therapy and the introduction of beam-scanning techniques. These should further increase the degree of dose conformity.

In the foreseeable future, the use of protons will remain limited to major centers only. Proton radiation therapy has provided a standard of long-term tumor control with acceptable morbidity rates. Most skull base centers either have GKN or LINAC-based systems available and/or modern three-dimensional conformal radiotherapy (intensity-modulated radiation therapy) capabilities. It is the hope of the author that other centers will combine their efforts in prospective trials to evaluate the benefits of radiosurgery or stereotactic fractionated photon radiotherapy techniques. Early results of stereotactic fractionated radiotherapy as reported by Debus, et al., ${ }^{9}$ are encouraging. Additional data on local tumor control following either GKS or LINAC radiosurgery are expected to be published soon to add to the available data reported by the Pittsburgh group. ${ }^{16}$

A very interesting development is the use of carbon ion therapy at the Heavy Ion Research Facility in Germany. Carbon ion therapy combines the physical advantages of protons with the differential increase in biological effectiveness of particles in tumors compared with normal tissues. In a preliminary analysis of the first 13 patients with skull base chordomas and chondrosarcomas in whom this therapy was applied, the authors did not find any evidence of increased acute toxicity. ${ }^{18}$

In summary, following a historically, almost fatalistic approach to skull base chordomas, recent refinements and new advances in microsurgical techniques, as well as introduction of various radiation therapy modalities, offer patients with skull base chordomas a realistic chance of long-term local tumor control and hopefully prolonged survival. Prognostic factors have been identified to guide 
physicians and patients in choosing the most appropriate management approach. Further developments are needed to improve the survival chances of patients in whom poor risk factors are present.

\section{References}

1. Austin JP, Urie MM, Cardenosa G, et al: Probable causes of recurrence in patients with chordoma and chondrosarcoma of the base of skull and cervical spine. Int J Radiat Oncol Biol Phys 25:439-444, 1993

2. Austin-Seymour M, Munzenrider J, Goitein M, et al: Fractionated proton radiation therapy of chordoma and low-grade chondrosarcoma of the base of the skull. J Neurosurg 70: 13-17, 1989

3. Benk V, Liebsch NJ, Munzenrider JE, et al: Base of skull and cervical spine chordomas in children treated by high-dose irradiation. Int J Radiat Oncol Biol Phys 31:577-581, 1995

4. Berson AM, Castro JR, Petti P, et al: Charged particle irradiation of chordoma and chondrosarcoma of the base of skull and cervical spine: the Lawrence Berkeley Laboratory experience. Int J Radiat Oncol Biol Phys 15:559-565, 1988

5. Borba LA, Al-Mefty O, Mrak RE, et al: Cranial chordomas in children and adolescents. J Neurosurg 84:584-591, 1996

6. Castro JR, Petti PL, Blakely EA, et al: Particle radiation therapy, in Leibel SA, Phillips TL (eds): Textbook of Radiation Oncology. Philadelphia: WB Saunders, 1998, pp 1223-1240

7. Catton C, O'Sullivan B, Bell R, et al: Chordoma: long-term follow-up after radical photon irradiation. Radiother Oncol 41: 67-72, 1996

8. Cummings BJ, Hodson DI, Bush RS: Chordoma: the results of megavoltage radiation therapy. Int J Radiat Oncol Biol Phys 9:633-642, 1983

9. Debus J, Hug EB, Liebsch NJ, et al: Brainstem tolerance to conformal radiotherapy of skull base tumors. Int J Radiat Oncol Biol Phys 39:967-975, 1997

10. Hug EB, Loredo LN, Slater JD, et al: Proton radiation therapy for chordomas and chondrosarcomas of the skull base. J Neurosurg 91:432-439, 1999
11. Hug EB, Slater JD: Proton radiation therapy for chordomas and chondrosarcomas of the skull base. Neurosurg Clin N Am 11: 627-638, 2000

12. Hug EB, Sweeney RS, Fuss M, et al: Proton radiation therapy in the management of pediatric base of skull tumors. Int $\mathbf{J}$ Radiat Oncol Biol Phys 48 (Suppl):181, 2000 (Abstract)

13. Munzenrider JE, Austin-Seymour M, Blitzer PJ, et al: Proton therapy at Harvard. Strahlentherapie 161:756-763, 1985

14. Munzenrider JE, Liebsch NJ: Proton therapy for tumors of the skull base. Strahlenther Onkol 175 (Suppl 2):57-63, 1999

15. Munzenrider JE, Liebsch NJ, Efird JT: Chordoma and chondrosarcoma of skull base: treatment with fractionated x-ray and proton radiotherapy, in Johnson JT, Didolkar MS (eds): Head and Neck Cancer. New York: Elsevier Science, 1993, Vol 3, pp 649-654

16. Muthukumar N, Kondziolka D, Lunsford LD, et al: Stereotactic radiosurgery for chordoma and chondrosarcoma: further experiences. Int J Radiat Oncol Biol Phys 41:387-392, 1998

17. Rich TA, Schiller A, Suit HD, et al: Clinical and pathologic review of 48 cases of chordoma. Cancer 56:182-187, 1985

18. Schulz-Ertner D, Debus J, Haberer T, et al: Heavy ion therapy in the treatment of skull base tumors: acute radiation induced toxicity. Int J Radiat Oncol Biol Phys 45 (Suppl):329-330, 1999 (Abstract)

19. Tai PT, Craighead P, Bagdon F: Optimization of radiotherapy for patients with cranial chordomas. Cancer 75:749-756, 1995

20. Verhey LJ, Smith V, Serago CF: Comparison of radiosurgery treatment modalities based on physical dose distributions. Int J Radiat Oncol Biol Phys 40:497-505, 1998

Manuscript received February 10, 2001.

Accepted in final form February 19, 2001.

Figs. 1 and 2 were previously published in the Journal of Neurosurgery, Volume 91, pages 432-439.

Address reprint requests to: Eugen B. Hug, M.D., Dartmouth Hitchcock Medical Center, Norris Cotton Cancer Center, One Medical Center Drive, Lebanon, New Hampshire 03756. email: Eugen.B.Hug@Hitchcock.ORG. 